\title{
Evolução funcional após discectomia endoscópica lombar, uma avaliação mais precoce de 32 casos*
}

\section{Functional Evolution after Percutaneous Endoscopic Lumbar Discectomy, an Earlier Evaluation of 32 Cases}

\author{
Bruno César Aprile ${ }^{10}$ Marcelo Campos Moraes Amato ${ }^{2}$ Cézar Augusto de Oliveira ${ }^{1}$ \\ ${ }^{1}$ Departamento de Coluna, Clínica SOU, São Paulo, SP, Brasil \\ 2 Departamento de Coluna, Instituto Amato, São Paulo, SP, Brasil

\begin{abstract}
Endereço para correspondência Bruno César Aprile, MD, Clínica SOU, R. Arcipreste Andrade 727, cj 145, Ipiranga, São Paulo, SP, 04268-020, Brasil (e-mail: dr.brunoaprile@gmail.com).
\end{abstract}

Rev Bras Ortop 2020;55(4):415-418.

\section{Resumo \\ Palavras-chave \\ - coluna vertebral \\ - deslocamento do disco invertebral \\ - avaliação de resultados (cuidados de saúde) \\ - endoscopia}

Objetivo Determinar o tempo de alta hospitalar e o período de melhora funcional e da dor dos pacientes submetidos a discectomia endoscópica percutânea lombar (DEPL).

Métodos Avaliação retrospectiva do tempo de internação e dos questionários escala visual análoga (EVA), índice de incapacidade Oswestry (IIO), e Roland-Morris de 32 pacientes submetidos a DEPL nos períodos pré-operatório e com 2 dias, e 1, 2, 4, 6, e 12 semanas pós-operatórias.

Resultados Todos os pacientes receberam alta em menos de 6 horas houve melhora estatística entre o período pré-operatório e 2 dias pós-operatório , sendo o valor médio do questionário EVA axial de 6,63 para 3,31, do EVA irradiado de 6,66 para 2,75, do IIO de $44,59 \%$ para $33,17 \%$ e do Roland-Morris de 14,03 para 10,34 . Tal diferença apresentou melhora progressiva até 12 semanas em todos os questionários. O IIO atingiu valores de incapacidade mínima (19,39\%) com 6 semanas de avaliação.

Conclusão Todos os 32 pacientes receberam alta hospitalar em até 6 horas. Houve melhora significativa dos sintomas funcionais e de dor já com 48 horas, apresentando ainda melhora adicional significativa e progressiva até o $3^{\circ}$ mês.

\section{Abstract \\ Objective To determine time period for hospital discharge and pain and function improvement in patients submitted to percutaneous endoscopic lumbar discectomy (PELD). Methods Retrospective evaluation of length of stay and visual analog scale (VAS), Oswestry disability index (ODI), and Roland-Morris questionnaire results in 32 patients undergoing PELD at the preoperative period and at 2 days and 1, 2, 4, 6 and 12 postoperative weeks. \\ Results All patients were discharged in less than 6 hours. There was a statistically significant improvement between the results obtained before the procedure and 2 days}

* Trabalho desenvolvido na Clínica SOU e no Instituto Amato,

São Paulo, SP, Brasil.

recebido

14 de Junho de 2019

aceito

15 de Agosto de 2019
DOI https://doi.org/

10.1055/s-0039-3402473. ISSN 0102-3616.
Copyright $\odot 2020$ by Sociedade Brasileira License terms de Ortopedia e Traumatologia. Published by Thieme Revinter Publicações Ltda, Rio de Janeiro, Brazil 


\section{Keywords}

- vertebral spine

- intervertebral disc dislocation

- outcome evaluation (healthcare)

- endoscopy postsurgery: the mean VAS for axial pain went from 6.63 to 3.31, the VAS for irradiated pain went from 6.66 to 2.75 , the Oswestry score went from 44.59 to $33.17 \%$, and the Roland-Morris score went from 14.03 to 10.34 . This difference progressively improved up to 12 weeks in all questionnaires. Regarding the Oswestry score, minimum disability values (19.39\%) were observed at 6 weeks.

Conclusion All 32 patients were discharged within 6 hours. Pain and function improved significantly after 48 hours, with further significant and progressive improvement until the $3^{\text {rd }}$ month.

\section{Introdução}

O tratamento padrão para hérnia discal lombar sintomática sem instabilidade é a descompressão por microdiscectomia lombar (MDL). ${ }^{1}$ Buscando uma menor agressão aos tecidos, foram desenvolvidas outras técnicas, tais como a discectomia endoscópica percutânea lombar (DEPL), sobre a qual foram conduzidos estudos que mostram evidência suficiente equiparando sua segurança e efetividade a médio e longo prazo em relação à MDL. ${ }^{2-4}$ Ainda não há consenso ou comprovação científica definitiva de superioridade ou não desta técnica sobre a MDL. Quando comparados os desfechos primários (melhora da dor, incidência de complicações e resultados funcionais) a médio e longo prazo ( 6 meses e mais de 2 anos), há estudos com resultados similares ${ }^{5,6}$ e outros demonstrando superioridade da DEPL sobre a MDL, tanto nos desfechos primários ${ }^{7-9}$ quanto nos secundários (tempo de cirurgia, de internação, e volume de sangramento). ${ }^{10,11}$ Apoiados em estudos com avaliação inicial mais precoce, ${ }^{12,13}$ elaboramos um protocolo de avaliação dos pacientes com início muito mais precoce do que a grande maioria dos estudos publicados sobre DEPL, iniciando 48 horas após a cirurgia, enquanto a primeira avaliação habitualmente se dá entre 4 a 6 semanas. Uma vez que a técnica já apresenta literatura comprovando sua segurança e eficácia a médio e longo prazos, ${ }^{14-18}$ buscamos entender quando realmente se inicia a melhora clínica, o que pode ser um fator determinante na escolha entre técnicas cirúrgicas até então similares em seus resultados.

\section{Materiais e Métodos}

Avaliamos retrospectivamente os dados coletados prospectivamente de 32 pacientes submetidos consecutivamente a DEPL. Todos apresentavam hérnia discal lombar única, sintomática por mais de 3 meses, com ou sem radiculopatia, e com falha do tratamento conservador. Todos foram submetidos a cirurgias eletivas, por via interlaminar ou transforaminal (nos casos com discopatias em topografia foraminal) e foram operados em clínicas e hospitais privados ou conveniados, em São Paulo, SP, entre 2014 e 2017, sendo submetidos a analgesia pósoperatória com nimesulida $100 \mathrm{mg}$ de 12/12 horas por 7 dias, dipirona 1 grama de $8 / 8$ horas por 10 dias e cloridrato de tramadol $50 \mathrm{mg}$ até de $8 / 8$ horas conforme a necessidade.

Foram excluídos pacientes com espondilolistese, cirurgias prévias lombares, e estenose de canal medular.
Além do momento de alta hospitalar, avaliamos os questionários escala visula análoga (EVA) (axial e irradiado), índice de incapacidade Oswestry (IIO), e Roland-Morris, que foram registrados no pré-operatório, com 2 dias, e com 1, 2, 4, 6, e 12 semanas de pós-operatório.

O trabalho teve a aprovação do comitê de ética da instituição sob o número CAAE 90766918.8.0000.5449.

\section{Resultados}

Incluímos 21 homens e 11 mulheres $(N=32)$ com idade entre 22 e 71 anos (média de 39,8 anos). Os resultados médios de cada questionário estão representados nas -Figuras $\mathbf{1}, \mathbf{2}$ e $\mathbf{3}$ e na - Tabela 1.

A média dos resultados apresentou redução estatisticamente significativa já entre o pré-operatório e o pós-operatório de 2 dias, com EVA axial variando de 6,63 para 3,31; EVA irradiado de 6,66 para 2,75; IIO de 44,59 para 33,17\%; e RolandMorris de 14,03 para 10,34. Tal diferença apresentou melhora progressiva até 12 semanas de pós-operatório. O IIO atingiu valores de incapacidade mínima (19,39\%) com 6 semanas.

Na comparação entre 2 dias e 12 semanas, a média dos resultados apresentou redução estatisticamente significativa no EVA axial, que variou de 3,31 para 1,94 ; IIO de $33,17 \%$ para $14,31 \%$, e Roland-Morris de 10,34 para 3,97 ; somente o

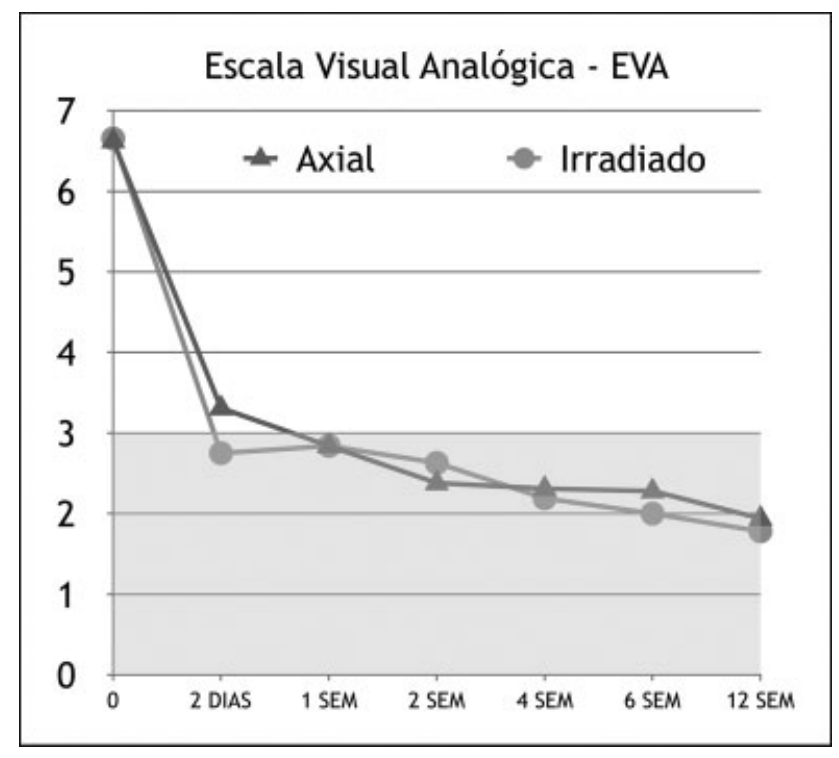

Fig. 1 Escala visual análoga. 


\section{OSWESTRY}

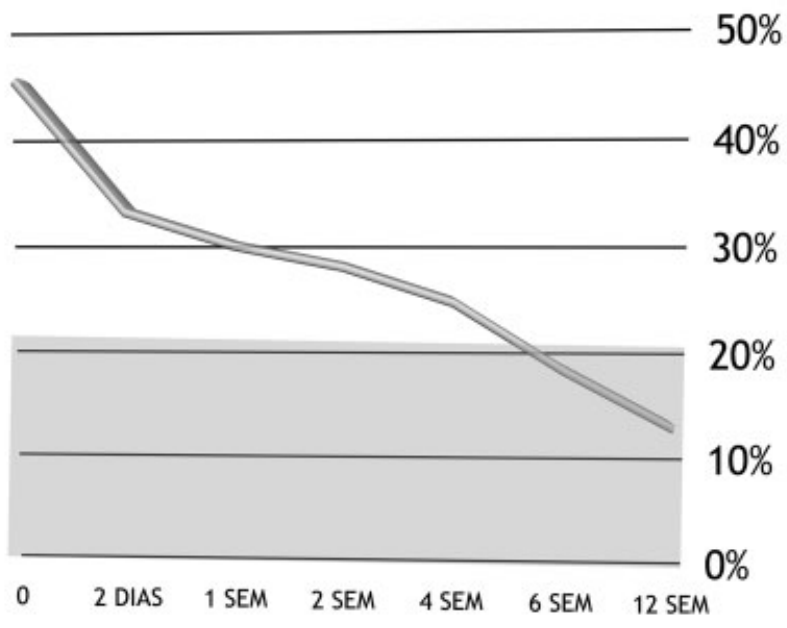

Fig. 2 Questionário Oswestry.

questionário EVA irradiado não apresentou diferença estatística nesse período, com variação de 2,75 para 1,78 .

Todos os pacientes receberam alta em até 6 horas. Não houve reinternação ou cirurgia complementar. Dois pacientes (6,45\%) apresentaram reherniação discal sem déficit neurológico; ambos decidiram por tratamento conservador, com melhora progressiva.

Não houve casos de sangramento significativo, lesão dural, ou déficit neurológico motor. Houve um caso (3,13\%) de cefaleia ortostática sem lesão dural identificada durante o procedimento, com resolução em 6 semanas, sem fístula ou

\section{ROLAND MORRIS}

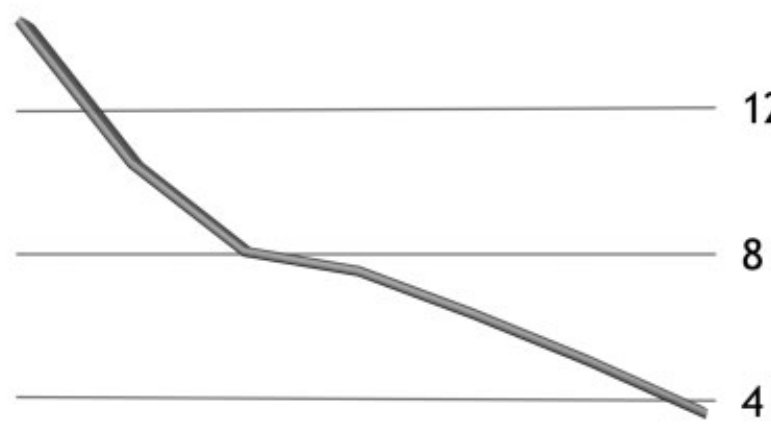

0 2 DIAS 1 SEM 2 SEM 4 SEM 6 SEM 12 SEM

Fig. 3 Questionário Roland-Morris.
Tabela 1 Valores médios dos questionários aplicados

\begin{tabular}{|l|l|l|l|l|}
\hline & $\begin{array}{l}\text { EVA } \\
\text { AXIAL }\end{array}$ & $\begin{array}{l}\text { EVA } \\
\text { IRRADIADO }\end{array}$ & Oswestry & $\begin{array}{l}\text { Roland- } \\
\text { Morris }\end{array}$ \\
\hline 0 & 6.63 & 6.66 & 44.59 & 14.03 \\
\hline 2 DIAS & 3.31 & 2.75 & 33.17 & 10.34 \\
\hline 1 SEMANA & 2.84 & 2.84 & 30.22 & 8.06 \\
\hline 2 SEMANAS & 2.38 & 2.63 & 28.38 & 7.56 \\
\hline 4 SEMANAS & 2.31 & 2.19 & 25.31 & 6.47 \\
\hline 6 SEMANAS & 2.28 & 2 & 19.39 & 5.28 \\
\hline 12 SEMANAS & 1.94 & 1.78 & 14.31 & 3.97 \\
\hline
\end{tabular}

coleção liquórica. Houve um caso $(3,13 \%)$ de disestesia no dermátomo operado, resolvida em 3 semanas com medicação e fisioterapia.

Para análise estatística dos resultados, utilizamos o software Minitab 18.1 (Minitab, Inc., State College, PA, USA) com análise de variância em modelo linear generalizado, e foram realizadas comparações pareadas de Tukey na variável tempo. o agrupamento foi feito utilizando o método de Tukey e índice de confiança de 95\%.

\section{Discussão}

Entre as cirurgias minimamente invasivas da coluna, a DEPL apresenta vantagens como a possiblidade de realização sob anestesia local, preservação das estruturas paraespinhais, e dor pós-operatória mínima. ${ }^{19}$ Apesar destas características favorecerem alta precoce e recuperação mais rápida, os estudos anteriores iniciavam suas avaliações apenas após 4 ou 6 semanas da cirurgia, perdendo a chance de avaliar se haveria melhora dos sintomas antes desse prazo, um dos reais benefícios do método. ${ }^{14-18}$

A melhora observada no presente estudo, comparando-se o pré-operatório com pós-operatório de 12 semanas tanto nos questionários de dor quanto nos questionários funcionais é compatível com as séries descritas na literatura. ${ }^{19,20}$ Choi et al. ${ }^{4}$ mostraram melhora dos parâmetros a partir do $1^{\circ}$ mês. No entanto, é inédita a confirmação da redução significativa dos valores dos questionários de dor e funcionais desde o pós-operatório de 2 dias, demostrando assim uma melhora ainda mais precoce dos sintomas com a DEPL.

Na revisão da literatura de Birkenmaier et al., ${ }^{11}$ os autores consideraram apenas a última avaliação funcional, com 2 anos ou mais de seguimento. Outros autores ${ }^{17}$ não especificam o momento em que os questionários foram respondidos. Portanto, apesar de existirem trabalhos demonstrando a superioridade da DEPL em relação a MDL nos quesitos menor lesão tecidual, ${ }^{7}$ melhora mais expressiva da dor e menor índice de complicações, ${ }^{8}$ menor tempo operatório, e maior

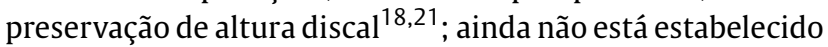
em qual momento os pacientes apresentam melhora da dor e nem qual o tempo necessário para alta hospitalar, com resultados conflitantes nos trabalhos publicados. Com este trabalho confirmamos que a melhora na DEPLé mais precoce do que os estudos geralmente demonstram. 
A melhora mais precoce se deu nos questionários de dor, e só depois nos funcionais, podendo ser resultado do receio pós-operatório, tanto dos pacientes como dos médicos, em retornar às atividades habituais, pelo risco de reherniação discal.

A alta hospitalar observada em menos de 6 horas para $100 \%$ dos casos é dado condizente com a descrição da técnica de DEPL como uma cirurgia ambulatorial. A maioria dos trabalhos descreve a alta como precoce, mas falha em não quantificar o momento exato da alta hospitalar. Lee Choi et al., ${ }^{19}$ em 2009, descreveram uma série de 25 pacientes submetidos a DEPL cuja permanência hospitalar média foi de 0,9 dia, enquanto que outras séries apresentaram alta mais tardia do que a da nossa série. ${ }^{22} \mathrm{~A}$ literatura mostra taxas de 0 a $11,1 \%{ }^{23}$ de recorrência e até $20,9 \%$ de necessidade de reoperação, ${ }^{4}$ e nossa série mostrou condizentes $6,45 \%$ de recorrência, com $0 \%$ de reoperação

\section{Conclusões}

Todos os 32 pacientes desta série receberam alta hospitalar em até 6 horas do término do procedimento. Os pacientes apresentam melhora significativa dos sintomas funcionais e de dor já com 48 horas de pós-operatório. A melhora álgica e funcional apresentou melhora adicional até as 12 semanas de pós-operatório. Na presente série, houve melhora funcional e da dor mais precoce do que a descrita na literatura para os pacientes submetidos a DEPL

\section{Conflito de Interesses}

Os autores declaram não haver conflito de interesses.

\section{Referências}

1 Botelho RV, Canto FT, Carvalho MV, et al. Diretrizes Clínicas na Saúde Suplementar - Hérnia de disco Lombar no Adulto: Tratamento Cirúrgico. São Paulo: Sociedade Brasileira de Ortopedia e Traumatologia e Sociedade Brasileira de Neurocirurgia; 2011

2 Peng CWB, Yeo W, Tan SB. Percutaneous endoscopic lumbar discectomy: clinical and quality of life outcomes with a minimum 2 year follow-up. J Orthop Surg Res 2009;4:20

3 Ruetten S, Komp M, Merk H, Godolias G. Full-endoscopic interlaminar and transforaminal lumbar discectomy versus conventional microsurgical technique: a prospective, randomized, controlled study. Spine 2008;33(09):931-939

4 Choi KC, Kim JS, Park CK. Percutaneous Endoscopic Lumbar Discectomy as an Alternative to Open Lumbar Microdiscectomy for Large Lumbar Disc Herniation. Pain Physician 2016;19(02):E291-E300

5 Phan K, Xu J, Schultz K, et al. Full-endoscopic versus micro-endoscopic and open discectomy: A systematic review and meta-analysis of outcomes and complications. Clin Neurol Neurosurg 2017;154(01): 1-12

6 Ruan W, Feng F, Liu Z, Xie J, Cai L, Ping A. Comparison of percutaneous endoscopic lumbar discectomy versus open lumbar microdiscectomy for lumbar disc herniation: A meta-analysis. Int J Surg 2016;31:86-92

7 Pan L, Zhang P, Yin Q. Comparison of tissue damages caused by endoscopic lumbar discectomy and traditional lumbar discectomy: a randomised controlled trial. Int J Surg 2014;12(05):534-537
8 Feng F, Xu Q Yan F, et al. Comparison of 7 Surgical Interventions for Lumbar Disc Herniation: A Network Meta-analysis. Pain Physician 2017;20(06):E863-E871

9 Ahn SS, Kim SH, Kim DW, Lee BH. Comparison of Outcomes of Percutaneous Endoscopic Lumbar Discectomy and Open Lumbar Microdiscectomy for Young Adults: A Retrospective Matched Cohort Study. World Neurosurg 2016;86:250-258

10 Li X, Han Y, Di Z, et al. Percutaneous endoscopic lumbar discectomy for lumbar disc herniation. J Clin Neurosci 2016;33:19-27

11 Birkenmaier C, Komp M, Leu HF, Wegener B, Ruetten S. The current state of endoscopic disc surgery: review of controlled studies comparing full-endoscopic procedures for disc herniations to standard procedures. Pain Physician 2013;16(04):335-344

12 Casimiro M. Short-Term Outcome Comparison Between FullEndoscopic Interlaminar Approach and Open Minimally Invasive Microsurgical Technique for Treatment of Lumbar Disc Herniation. World Neurosurg 2017;108:894-900.e1

13 Li M, Yang H, Yang Q. Full-Endoscopic Technique Discectomy Versus Microendoscopic Discectomy for the Surgical Treatment of Lumbar Disc Herniation. Pain Physician 2015;18(04):359-363

14 Kapetanakis S, Giovannopoulou E, Charitoudis G, Kazakos K. Health-related quality of life (HRQoL) following transforaminal percutaneous endoscopic discectomy (TPED) for lumbar disc herniation: A prospective cohort study - early results. J Back Musculoskeletal Rehabil 2017;30(06):1311-1317

15 Chen HT, Tsai CH, Chao SC, et al. Endoscopic discectomy of L5-S1 disc herniation via an interlaminar approach: Prospective controlled study under local and general anesthesia. Surg Neurol Int 2011;2:93

16 Porchet F, Bartanusz V, Kleinstueck FS, et al. Microdiscectomy compared with standard discectomy: an old problem revisited with new outcome measures within the framework of a spine surgical registry. Eur Spine J 2009;18(Suppl 3):360-366

17 Li XC, Zhong CF, Deng GB, Liang RW, Huang CM. Full-Endoscopic Procedures Versus Traditional Discectomy Surgery for Discectomy: A Systematic Review and Meta-analysis of Current Global Clinical Trials. Pain Physician 2016;19(03):103-118

18 Markovic M, Zivkovic N, Ruetten S, Ozdemir S, Goethel D. Fullendoscopic interlaminar versus microsurgical operations in lumbar compressive lesions surgery: prospective randomized trial of overall 570 patients. J Pain Relief 2017;6(06):1-7

19 Lee DY, Shim CS, Ahn Y, Choi YG, Kim HJ, Lee SH. Comparison of percutaneous endoscopic lumbar discectomy and open lumbar microdiscectomy for recurrent disc herniation. J Korean Neurosurg Soc 2009;46(06):515-521

20 Ruetten S, Komp M, Merk H, Godolias G. Full-endoscopic cervical posterior foraminotomy for the operation of lateral disc herniations using 5.9-mm endoscopes: a prospective, randomized, controlled study. Spine 2008;33(09):940-948

21 Lee SH, Chung SE, Ahn Y, Kim TH, Park JY, Shin SW. Comparative radiologic evaluation of percutaneous endoscopic lumbar discectomy and open microdiscectomy: a matched cohort analysis. Mt Sinai J Med 2006;73(05):795-801

22 Righesso O, Falavigna A, Avanzi O. Comparison of open discectomy with microendoscopic discectomy in lumbar disc herniations: results of a randomized controlled trial. Neurosurgery 2007;61 (03):545-549, discussion 549

23 Matsumoto M, Watanabe K, Hosogane N, et al. Recurrence of lumbar disc herniation after microendoscopic discectomy. J Neurol Surg A Cent Eur Neurosurg 2013;74(04):222-227

24 Tenenbaum S, Arzi H, Herman A, et al. Percutaneous posterolateral transforaminal endoscopic discectomy: clinical outcome, complications, and learning curve evaluation. Surg Technol Int 2011;21:278-283 